\title{
Universidades vs terciarização do ensino superior: a lógica da expansão do acesso com manutenção da desigualdade: 0 caso brasileiro'
}

ANTÔNIO AUGUSTO PEREIRA PRATES*

\section{A revolução dos números}

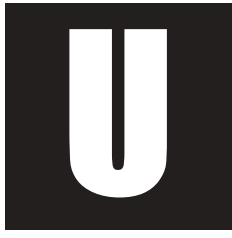

m dos traços mais marcantes do cenário social no sec. XX foi a forte tendência, desde o primeiro quartel do século, de ampliação do acesso ao ensino superior tanto na Europa, quanto nos EUA. Embora não se tenha, ainda hoje, uma única teoria que possa explicar de forma definitiva a que se deveu essa expansão do sistema de ensino superior na sociedade moderna, podem ser sugeridos alguns fatores que, certamente, contribuíram para essa expansão. K. H. Jarusch (1983) aponta quatro dimensões, relacionadas, respectivamente, aos enfoques econômico, sociológico, político e cultural, como elementos, senão suficientes, indispensáveis para a compreensão deste fenômeno. ${ }^{2}$

O enfoque econômico privilegia o argumento de que a sociedade industrial "madura" requer uma força de trabalho mais profissionalizada e educacionalmente credenciada, especialmente na área de administração

\footnotetext{
* Mestre em Sociologia pela State University of New York at Stony brook e Doutor em Sociologia pela UFMG. Professor Adjunto IV do Dpartamento de Sociologia e Antropologia da FAFICH-UFMG. Brasil.

1 Este artigo foi extraído da Tese de doutoramento do autor denominada "Os Sistemas de Ensino Superior na Sociedade Contemporânea: Diversificação, democratização e Gestão organizacional O caso brasileiro" apresentada na UFMG, 2005.

2 O texto a seguir, sobre essas dimensões, é baseado, no texto citado de K.H. Jarausch, 1983: 26.
} 
pública e privada. Ao mesmo tempo, estas características da sociedade implicam maior prosperidade e abertura dos canais de mobilidade, tornando a educação um bem de consumo para alguns setores da população. De outro lado, o segmento mais tecnológico da indústria como o setor eletrônico e químico demanda mais pesquisa aplicada e, conseqüentemente, maior contato direto com a Universidade.

O enfoque sociológico direciona seu olhar, de um lado, para o surgimento da "nova classe média", buscando na educação os degraus universalistas de mobilidade, e de outro, para a pressão dos membros da plutocracia que, sofrendo o esvaziamento dos critérios adscritos de status, são empurrados ladeira-abaixo para a "velha" classe média e buscam, portanto, assegurar sua posição de status via credenciais educacionais.

O argumento político enfatiza a emergência de políticas governamentais, buscando incorporar setores "marginalizados" na sociedade industrial, como o proletariado na virada do séc. XIX e, também, a expansão dos serviços públicos acompanhando a consolidação do papel normativo do estado-racional demandando profissionais graduados, como bem ilustra o caso da Alemanha.

Finalmente, o enfoque culturalista que, partindo do pressuposto do apelo atraente do ideal do "ser humano educado", enfatiza a busca popular incessante para o auto-aprimoramento, sem nenhuma necessidade de justificativa funcional.

Entretanto, se estes fatores desempenharam, e continuam a desempenhar, um papel relevante no desencadeamento da abertura do sistema de ensino superior na sociedade moderna, foi somente a partir da década de 60 que se observou uma verdadeira revolução de números neste nível do sistema educacional. Ainda assim, de acordo com o índice de classificação do grau de "abertura" do sistema de ensino superior de Martin Trow $(1975)^{3}$, pode-se afirmar que, em 1996, apenas 6 países (Coréia , Canadá,

3 Martin Trow (1970) sugeriu uma tipologia de classificação de sistemas de ensino superior com três categorias: sistema de elite, taxa líquida de escolarização até 15\%, sistema de massa, taxa líquida de 15 a 33\% e sistema universal com uma taxa líquida de 33 a $40 \%$. 
Bélgica, Grécia, França e Estados Unidos) estavam na categoria de "sistema universal" e 10 países ainda se encontravam na categoria de "sistema de elite" ( entre eles, Suécia, Hungria, Alemanha, Dinamarca, Suíça, Brasil). ${ }^{4}$ É inegável, contudo, que a partir dos anos 60 , iniciou-se um processo de crescente abertura do sistema de terceiro grau em todo o mundo levando à transformação, na maioria dos países, dos seus sistemas de ensino superior: de um modelo de "elite" para um modelo de "massa". ${ }^{5}$ O número de estudantes na educação superior, em 1995, no mundo inteiro, era de 82 milhões de estudantes. Em 1960, este número era de 13 milhões, em 1970, este número dobrou, 26 milhões e, em 1980, o número de estudantes de nível terciário já atingia a casa dos 51 milhões. Portanto, de 1960 a 1995 o número de estudantes de educação superior no planeta cresceu mais de seis vezes. ${ }^{6}$ R. Boudon (1981:5) reafirma esta tendência de universalização do ensino superior nas sociedades industriais,

... na maioria das sociedades industriais, a desigualdade das oportunidades perante o ensino diminuiu de modo lento e regular no curso das últimas décadas. Em outras palavras, a probabilidade de um adolescente saído das classes inferiores de chegar, por exemplo, à universidade aumentou em tempo mais rápido que a mesma probabilidade em relação a um adolescente saído das classes superiores. As ordens de grandeza permanecem diferentes, mas a tendência à atenuação das disparidades mostra-se não negligenciável e universal no conjunto das sociedades industriais ocidentais.

Essa revolução numérica da ampliação do sistema de ensino superior no mundo inteiro teria sido impossível, caso o modelo tradicional das uni-

4 ver Nunes E.,Nogueira, A., Ribeiro, L. op. cit.:51.

5 Embora alguns países economicamente centrais, como a Inglaterra, tenham feito esta transformação somente na década de 80. (Mary Henkel, op. cit:30)

6 Dados extraídos do "World Statistical Outlook on Higher education: 1980-1995", UNESCO, 1998. 
versidades clássicas não desse lugar a outros mecanismos e formatos institucionais alternativos.

\section{Diferenciação institucional e expansão de vagas.}

O termo diferenciação institucional tem sido utilizado na literatura especializada para denominar um processo de diversificação funcional entre as instituições de ensino de nível terciário. Este processo de diferenciação estabelece papéis distintos para as "históricas" e clássicas instituições universitárias e para os mais recentes colleges e institutos de formação técnico-profissional vocacionalmente orientados. ${ }^{7}$ Embora essa diversificação do sistema "clássico" do ensino superior se tenha intensificado na segunda metade do séc. passado, tanto na Europa quanto nos EUA, de fato, ele vem ocorrendo desde meados do séc XIX em países como Alemanha, Inglaterra, Rússia e Estados Unidos. Como bem demonstra K. H. Jaraush (op.cit.) em seu estudo comparativo de sistemas de ensino superior entre esses quatro países no período de 1860-1930. De acordo com esse estudo, no final do séc. XIX e no início do séc. XX ocorreu um enorme crescimento relativo dos sistemas de ensino superior, através de um processo de diversificação institucional. Na Alemanha, as antigas politécnicas de nível secundário ( as Technishe Hochschulen) foram elevadas ao nível terciário em 1875; na Inglaterra instituições como os British Redbricks deixaram de ser centros de treinamento técnico avançados para se transformarem em instituições universitárias tradicionais. Nos EUA, os colleges vocacionais de 1 e 2 anos já dominavam quantitativamente o cenário do ensino terciário. Na Rússia, os

7 Este sistema de diversificação, quando polarizado entre o modelo univerisitário clássico e as instituições de formação técnica profissional, como ocorreu na Inglaterra, com a criação dos institutos Tecnológicos no início dos anos 60, ficou conhecido na literatura como "sistema binário". Nos anos 80, esses institutos na Inglaterra foram transformados em universidades. 
institutos tecnológicos substituíam as universidades tradicionais. Nas palavras de K. H. Jaraush ( op. cit.:19),

os mecanismos fundamentais em todos os quarto países parecem ser a adição de novos tipos, o upgrading das instituições secundárias existentes e a transformação de sua função na direção do ideal universitário tradicional.

Contudo foi a partir dos anos sessenta, do séc. passado, que o processo de diferenciação funcional adquiriu "universalidade" como resposta a, pelo menos, três tipos distintos de estímulos que emergiam no contexto das sociedades contemporâneas, quais sejam: a) a demanda de inclusão social; b) a demanda de formação mais rápida e mais sensível às necessidades da "nova economia" de mão-de-obra qualificada ${ }^{8}$ e c) a resistência à "abertura" das universidades do tipo "clássico". Ainda que, como veremos mais adiante, esse processo de diferenciação tivesse redundado em um sistema de estratificação de instituições de terceiro grau, marcadamente entre universidades de ensino e pesquisa, universidades de ensino e, na base da pirâmide, instituições de ensino técnico-profissional, sem dúvida foi esse processo que possibilitou o crescimento vertiginoso do acesso à educação superior em todo o planeta. Como afirmou Paul Ryan ( 2003:147), "expansão e vocacionalização têm andado de mãos dadas". ${ }^{9}$ De fato, é este tipo de mudança nos sistemas de ensino superior que tem produzido impactos relevantes, em termos de crescimento e flexibilização, sobre os sistemas de ensino superior, independentemente das várias nuances e conjunturas regionais, nacionais que, obviamente, medeiam a maneira de crescimento adaptando-o às suas peculiaridades.

8 Ver, M. Osborne, Increasing or Widening Participation in Higher Education? - a European view." In Eurppean Jornal Of Education, vol 38, no. 1, 2003; Paul Ryan, "Evaluating Vocationalism", in European Journal of Education, vol 38, no.2, 2003.

9 "expansion and vocationalisation have gone hand in hand" 
Como mostra A. Wagner (2000:12,13), as várias estratégias de diversificação institucional no contexto atual diferem entre países e regiões, de acordo com o modelo institucionalizado de ensino superior em cada país . Entre essas estratégias ele enumera:

- "O reforço de programas de nível-terciário, vocacionalmente orientados, oferecidos num distinto sub-sistema(do circuito universitário convencional) de instituições, na França, Finlândia, Alemanha, Austrália, Portugal, Suíça, Japão, Nova Zelândia e, em menor extensão, EUA, e em desenvolvimento na República Tcheca.

- O estímulo ao desenvolvimento de perfis distintos dentro de um mesmo e único sistema, como na Suécia, Austrália, Reino Unido, e generalizadamente nos EUA.

- A tranformação de programas/instituições de nível secundário para o nível terciário "profissional", como no caso da enfermagem na Austrália e Itália e do treinamento de professores de escolas primárias na França e outros países.

- A introdução de cursos universitários de curta duração, tais como os cursos com grau de bacharel na Dinamarca e na República Tcheca e sob consideração, para introdução e expansão, na França, Alemanha, e Itália ( entre outros países da Europa Continental).

- A provisão de condições favoráveis, ou mesmo suporte financeiro direto ou indireto, para o estabelecimento e desenvolvimento de programas específicos dentro de um setor privado paralelo, como no Japão, Koréia, EUA e Portugal, e em grau mais limitado na França e mais recentemente na Alemanha.

- A integração dentro dos programas de estudos das universidades convencionais de módulos vocacionalmente orientados, "estudos aplicados ou integrados" ou experiência de trabalho como no Reino Unido, EUA e França. 
- Experiências, com maior ou menor escopo, de expansão da participação em estudos de nível terciário através de instituições ou outros meios distintos das instituições formais de nível terciário, tais como TAFE Institutes na Austrália, faculdades de educação continuada no Reino Unido, vários cursos-livres de educação a distância e outras opções alternativas nos EUA (e outros países).

- Encorajamento ou permissão para cooperação através de segmentos e fronteiras, incluindo franchising no Reino Unido, articulações e arranjos específicos na Nova Zelândia e nos EUA, programas com junções de grau/diploma no Japão." ${ }^{10}$

\footnotetext{
10 "-reinforcing vocationally-oriented, tertiary-level programs offered in a distinct sub-system of institutions, in France, Finland, Germany, Australia, Portugal, Switzerland, Japan, New Zeland an to a lesser exten the United States, and in development in the Czech republic;

- stimulating the development of more distinct institutional profiles within a single system, as in Sweden, Australia, the United kingdom and generally the United States;

- upgrading from secondary to tertiary level "professional" programs or institutions, as in the case with nursing in Australia, and Italy and primary school teacher training in France and other countries; - introducing short university study programs, such as bachelor's degree studies, in Denmark and the Czech republic and under consideration for introduction and espansion in France, germany and italy (among other continental European countries);

- providing favorable conditions, if not direct or indirect financial support, for the establishment and development of distinctive programs within a parallel private sector, as in japan, the United States and Portugal, and to a much more limited degree in france and more recently in Germany;

- integrating within conventional university study programs vocationally-oriented work modules, " applied or integrated studies" or work experience, as in the Unitede Kingdom, United Statyes and Frqance;

- experiencing, with greater or lesser oversight, the expansion of participation in tertiary-level studies trough institutions or means other than formal tertiary education institutions, sucha as TAFE institues in Australia, futher education colleges in the United Kingdom, various free-standing distance education and other alternative mode options in the United States ( and other countries);

- encouraging or permitting cross-segment and cross-border or co-operation, to include franchising in the Unitede Kingdom, specific articulation arrangements in New Zeland and the United States, joint degree/diploma programs in Japan."
} 
Há, na literatura especializada, uma intensa discussão sobre estes processos de diversificação, se estão, ou não, relacionados à 'marketização' ou mercantilização do sistema de ensino superior. Guy Neave (2000), por exemplo, enfatiza a tendência de crescimento de um paradigma voltado para a lógica 'economicista' na qual os resultados do sistema educacional são vistos à luz da lógica da 'taxa de retorno', seja esta individual ou social. Neste paradigma, a questão da diversificação ou diferenciação programática ou institucional está a reboque de uma estratégia maior de mercantilização do ensino superior, tratado pelos policymakers como se fosse uma 'mercadoria'. Esta linha crítica de análise está também presente no estudo de Pedro Teixeira e Alberto Amaral(2001:361). Esses autores criticam a proposição de que "(...)freqüentemente é considerado que diversidade na educação superior é mais compatível com o livre jogo das forças de mercado" e, através de alguns estudos de caso na Europa, mostram que não há evidência suficiente para suportar esta hipótese. De outro lado, há os que, como Alan Wagner (1999) e C. Moura Castro ( 2001), sustentam a proposição de que, embora os processos de diversificação programática e institucional não garantam, por si só, a eliminação da iniqüidade no sistema educacional, os efeitos desses processos sobre o aumento dos canais de acesso ao ensino superior são positivos, e isso tem favorecido os grupos econômica e socialmente mais desprotegidos das sociedades industriais. Nesta mesma linha Martin Trow ( 1986:139.) sugere que

As novas Instituiçôes Européias - Instituts universitaires de technologie, Fach hochschulen, e outros - e os novos community collegfes e colleges públicos foram criados ou expandidos para atender vários propósitos: reduzir a pressão de matrícula sobre as universidades (...); prover formas alterantivas de instrução, livre das restrições das disciplinas e do corpo de professores, com mais espaço para a instrução sistemática e trabalho 
interdisciplinar, e frequentemente, embora nem sempre explicitamente, criar um conjunto de instituições livre das conexôes histórias com as classes altas, mais próximas ao mundo do trabalho, e assim com um clima intellectual mais atrativo aos estudantes da classe trabaIhadora. ${ }^{11}$ (grifo meu).

Mas o crescimento da expansão do sistema não tem sido suficiente para quebrar os "velhos" padrões socialmente estruturados de desigualdade. Como sugere o próprio A. Wagner(op.cit:16)

Embora a expansão tenha avançado em vários países como um meio de trazer para dentro da educação terciária aqueles que são sub-representados, diferenças persistem no 'mix' social e econômico dos estudantes. Enquanto tem aumentado a matrícula dos estudantes vindos de grupos de baixo status sócio-econômico, o mesmo tem ocorrido com aqueles vindos de grupos bem-representados na educação terciária ${ }^{12}$

A Tabela 1 ilustra as observações de A. Wagner.

Em todos os países considerados, a probabilidade de adquirir uma qualificação de terceiro grau varia de duas a seis vezes favoravelmente àqueles estudantes cujos pais completaram educação terciária, comparada com a daqueles cujos pais não terminaram o segundo grau. Mas, o que

11 "The new European institutions - Instituts universitaires de technologie, Fachhochschulen, and others - and the new state and community colleges in the United States were created or expandeded for a variety of purposes: to reduce enrollment pressures on the universities ( ...); to provide alternative forms of instruction, freed from constraints of the university chair and faculty system, with more scope for systematic instruction and interdisciplinary work; and often, though not always explicitly, to create a set of institutions free from historical links to the upper classes, closer to the world of work, and thus with an intellectual climate more attractive to working-class students"

12 "Although expansion has been advanced in several countries as a means to bring into tertiary education those who are under-represented, differences in the socialand economic mix (sic) of students persist. While enrollment of students from low social and economic groups have increased, so too thave thoser from groups already well-represented in tertiary education." 
mais chama a atenção é que, em seis países (Austrália, Canadá, Alemanha, Nova Zelândia, Polônia e Reino Unido), essa probabilidade aumente para a coorte jovem em relação à mais velha, ou seja, o sistema de educação superior nesses países tende a ser mais elitista hoje do que no passado. ${ }^{13}$ Este indicador torna-se ainda mais dramático se o olharmos à luz dos retornos de renda relativos ao nível educacional nesses países. A tabela abaixo mostra esta informação.

Como se pode observar, o ganho relativo de renda de emprego para quem atingiu o nível de educação terciária é bastante significativo, mas, para quem conseguiu atingir o nível terciário, em universidades, o ganho relativo, para a maioria dos países, é muito mais expressivo. Considerando, como temos mostrado, que o crescimento vertiginoso da educação superior nos países mais desenvolvidos desde os anos 70/80 deveu-se principalmente à expansão do ensino terciário de tipo não-universitário, ${ }^{14}$ pode-se constatar que os efeitos dessa expansão sobre a estrutura convencional de desigualdade produzida pelo grau de acesso à educação superior, não são tão significativos como se poderia esperar pelo aumento bruto da participação neste nível de ensino, porque o acesso às universidades continua sendo restrito às pequenas parcelas privilegiadas da população. K.H. Jarausch (op. cit: 22) aponta para a ocorrência deste fato desde o final do séc. XIX, nos países por ele estudados. De acordo com ele, a expansão do sistema de educação superior veio acompanhado de

13 Thomas J. Kane (1995) demonstra, ainda, que embora o Governo Federal Americano gaste 13 bilhões de dólares em subsídios à educação superior e os estados, 40 bilhões de dólares, o aumento de anuidades do sistema público, especialmente para o caso dos two year-colleges, produz efeito negativo sobre o volume de matrícula dos estudantes das famílias situadas no quartil de renda mais pobre.

14 Como afirma M. Trow, $(1986: 160,161)$ " On the whole, the pressure for equality across sectors hás constrained growth, trhough the cost of the new sectors and the deterrent of high academic standards. The determination of egalitarians in the new sectors to match the older sectors in academic standards and status, in order to provide the very best for the lower-class clientele, has surely inhibited the growth of mass higher education throughout western Europe." 
Tabela 1 - Aumento diferencial da probabilidade de aquisição de uma qualificação de nível terciário para indivíduos cujos pais tem educação terciária completa, comparados com indivíduos cujos pais não têm educação secundárai completa por faixa de idade e $\mathrm{p}$

\begin{tabular}{|c|c|c|c|}
\hline & Total (16-25) & Younger age (26-35) & Older age (46-55) \\
\hline Austrália & 2,0 & 2,4 & 1,9 \\
Bélgica & 3,3 & 2,6 & 4,4 \\
Canadá & 2,4 & 2,9 & 2,2 \\
Alemanha & 2,3 & 2,9 & 2,4 \\
Irlanda & 4,8 & $\mathrm{~m}$ & $\mathrm{~m}$ \\
Holanda & 3,3 & 3,3 & 4,3 \\
Nova Zelândia & 2,1 & 2,8 & 2 \\
Poland & 5,8 & 6,6 & $\mathrm{~m}$ \\
Sweden & 2,2 & 2,8 & 2,6 \\
Switzerland & 4,3 & 4,3 & 5,6 \\
Reino Unido & 2,9 & 3,3 & 3,1 \\
Estados Unidos & 3,3 & 3,6 & 4,6 \\
\hline
\end{tabular}

Fonte - Education at a Glance - OECD Indicators 1998, Paris. Wagner, A. op. cit: 18

centros competitivos de alta aprendizagem com algo distinto em sua missão educacional criaram uma hierarquia de status de tipos institucionais, os quais oferecem acesso social compensatório, mas também condenam os setores mais 'modernos' à persistente inferioridade, a menos que eles se conformem ao velho modelo neo-clássico ${ }^{15}$

Estudos mais recentes como o de Shavit e Blossefeld(1993) realizado em 11 países diferentes, mostram que, mesmo considerando sistemas educacionais distintos, a expansão deles não implica, necessariamente, o au-

15 "competing centers of higher learning with somewhat different educational missions created a status hierarchy of institutional types, which offered compensatory social Access butalso condemned the more 'modern` sectors to continuing inferiority unless they conformed to the older neo-classical mold." 
Tabela 2 - Retornos de renda de emprego relativos ao nível educacional de pessoas de 25-64 anos em países da OECD. (Nível mais alto de educação secundária $=100)$ - 1995*

\begin{tabular}{|c|c|c|c|}
\hline & $\begin{array}{l}\text { Abaixo da educação } \\
\text { secundária }\end{array}$ & \begin{tabular}{|} 
Educação terciária \\
não-universitária
\end{tabular} & $\begin{array}{c}\text { Educação } \\
\text { universitária }\end{array}$ \\
\hline $\begin{array}{c}\text { América do Norte } \\
\text { Canadá } \\
\text { Estados Unidos }\end{array}$ & $\begin{array}{l}87 \\
68\end{array}$ & $\begin{array}{l}110 \\
119\end{array}$ & $\begin{array}{l}156 \\
174\end{array}$ \\
\hline $\begin{array}{c}\text { Área do Pacífico } \\
\text { Austrália } \\
\text { Nova Zelândia }\end{array}$ & $\begin{array}{l}89 \\
82\end{array}$ & $\begin{array}{l}111 \\
106\end{array}$ & $\begin{array}{l}161 \\
142\end{array}$ \\
\hline $\begin{array}{c}\text { União Européia } \\
\text { Dinamarca } \\
\text { Finlândia } \\
\text { França } \\
\text { Alemanha } \\
\text { Irlanda (1993) } \\
\text { Itália } \\
\text { Holanda } \\
\text { Portugal } \\
\text { Suécia } \\
\text { Reino Unido }\end{array}$ & $\begin{array}{l}83 \\
93 \\
80 \\
78 \\
85 \\
77 \\
77 \\
68 \\
89 \\
75\end{array}$ & $\begin{array}{c}104 \\
126 \\
128 \\
111 \\
123 \\
\ldots \\
124 \\
\ldots \\
109 \\
132\end{array}$ & $\begin{array}{l}193 \\
187 \\
175 \\
163 \\
183 \\
194 \\
162 \\
183 \\
151\end{array}$ \\
\hline $\begin{array}{c}\text { Outros Países da OECD } \\
\text { República Tcheca } \\
\text { Noruega } \\
\text { Suíça } \\
\text { Média dos Países }\end{array}$ & $\begin{array}{l}66 \\
82 \\
67 \\
79\end{array}$ & $\begin{array}{l}\ldots \\
123 \\
145 \\
119\end{array}$ & $\begin{array}{l}158 \\
149 \\
157 \\
162\end{array}$ \\
\hline
\end{tabular}

*Fonte - World Statistical Outlookon Higher education: 1980-1995. Unesco: 1998.

mento da importância de critérios meritocráticos para o acesso a eles. (apud Fernandes, D. 1999:216).

É inegável, portanto, o dilema contemporâneo da criação de formas institucionais alternativas de ensino superior, baseadas na ênfase do treinamento técnico, ao invés do treinamento acadêmico, como condição básica 
de acesso a esse nível de ensino para as camadas da população de baixarenda, ao mesmo tempo em que segrega o acesso às universidades de prestígio às camadas mais privilegiadas da sociedade. ${ }^{16}$ Esse sistema de estratificação educacional, embora não comprometa o "esforço" igualitarista das políticas de expansão do ensino superior através da criação, no seu interior, dos setores "vocacionais", diminui bastante o impacto da expansão do sistema sobre a desigualdade de acesso por classe social.

\section{Caso brasileiro: expansão vs iniqüidade no sistema de ensino superior}

O modelo de expansão do ensino superior no Brasil deu-se principalmente via sistema privado. Em 2002, havia 1.637 instituições de ensino superior no País. Sendo que 195 eram públicas (73 federais) e 1.442 eram de natureza privada (1125 particulares e 317 Comunitárias, Confessionais ou Filantrópicas). Essas instituições ofereciam 14.399 cursos de graduação presenciais; desses, 5.252 são das instituições públicas (2.316 das federais) e 9.147 das instituições privadas.(INEP/MEC, Censo do Ensino Superior 2002).

De 1960 a 1980 o volume de matrículas cresceu um pouco mais de nove vezes nas instituições públicas (federais, estaduais e municipais),

16 Em 1999 foi acordado entre 29 países da Europa um documento denominado Declaração de Bolonha que, de forma geral, tentava uniformizar o sistema de ensino superior na Europa, através da criação de somente dois ciclos de estudo naquele nível: o primeiro ciclo, com, no mínimo, 3 anos de duração, equivaleria aos atuais cursos de nível de graduação, mas diferentemente destes, seriam mais voltados para a formação "vocacional", i.e, mais voltada para o mercado. O segundo ciclo seria o de pós-graduação, mestrado e doutorado, com duração aproximada de 5 anos, voltado para a orientação mais acadêmica e de pesquisa, no caso do doutorado. Esse sistema deveria facilitar a mobilidade de estudantes e professores entre universidades européias e, por outro lado, possibilitaria o atendimento das demandas de formação de curto-prazo, sem criar instituições especializadas em um ou outro ciclo de estudo, eliminando, assim, o caráter de estratificação entre os dois níveis de ensino. Ver, para uma apresentação mais completa do Processo Bolonha, www.esib.org, Bologne Process Committee, "Introduction to the Bologne Process" ( consulta realizada em 05/05/2005) 
enquanto no ensino privado este número foi mais de 21 vezes. ${ }^{17} \mathrm{O}$ volume total de matrículas nos dois sistemas, no período entre 1960 e 2002, mostra uma revolução numérica do crescimento da participação do setor privado no sistema global: em 1960 o setor privado respondia por 43,96\% da matrícula global no ensino superior no País e em 2002 essa participação cresceu para 69,77\%.

Em 2000, somente 7,37\% - cerca de 1.700 .000 estudantes - da coorte de 20 a 24 anos teve acesso à educação superior no país. ${ }^{18}$ Do ponto de vista do impacto do título universitário sobre o nível de renda, Schwartzman (2000) mostra que a educação superior no Brasil aumenta o rendimento relativo das pessoas em 3,59 vezes mais do que recebem aqueles com ensino médio completo. Para os países da OECD, este multiplicador oscila entre 1,5 e 1,8. Ainda de acordo com Schwartzman (2000:2,5), "este diferencial de renda é ainda mais significativo porque está associado a uma mobilidade social muito grande experimentada por estes estudantes."

Este quadro aponta para um enorme fosso de iniqüidade produzido pelo baixo acesso da população ao ensino superior na sociedade brasileira. Este fosso torna-se ainda mais dramático quando são olhadas as informações sobre as diferenças internas do grupo "privilegiado" com acesso a esse nível de ensino no Brasil. Os dados mostram que $72 \%$ dos estudantes desse nível de ensino estava na População Economicamente Ativa (PEA) em 1997, e cerca de 54\% deles estudava à noite (Schwartzman, S.1998:5). Em 2002, como mostram os dados do censo de ensino superior do INEP, 58\% dos estudantes de ensino superior no país continuavam no ensino noturno, sendo que, destes, $18,8 \%$ estavam no sistema público e somente $6,5 \%$ nas Federais, ou seja, a grande maioria, 74,7\%, dos estudantes brasileiros mais

17 Fonte: MEC/SEDIAE/SEEC - Sinopse Estatística do Ensino Superior2001, 2002;

$18 \mathrm{SESu} / \mathrm{MEC}$, Documento Interno, s/d. 
pobres, os que trabalham, estudam em escolas privadas cujo ensino é, em geral, de pior qualidade, se comparado com o das IFES. ${ }^{19}$

Um estudo recente de Ricardo P. de Barros e Miguel N. Foguel (2001) constroem um índice para medir a concentração do acesso ao ensino superior público no Brasil, que denominam de Índice Quase-Gini. ${ }^{20}$ De acordo com esse índice, o nível de concentração do acesso ao ensino superior público brasileiro é maior do que a concentração da distribuição da renda familiar no País, que já é considerada das mais altas em termos mundiais.

A questão da iniqüidade do sistema de ensino superior do País pode, ainda, ser mais bem qualificada tomando-se em consideração os dados que mostram seus efeitos sobre o status sociocupacional.

19 Num estudo sobre a questão da equidade no sistema de ensino superior brasileiro, os pesquisadores Sampaio, M.H, Limongi, F. e Torres H. (2000) afirmam de forma equivocada, a nosso ver, que "a visão difundida na opinião pública é que o sistema de ensino superior brasileiro se caracteriza por uma seleção altamente perversa, segundo a qual, estudantes com renda familiar elevada estudam em estabelecimentos de ensino superior públicos, enquanto estudantes provenientes de famílias de baixa renda familiar pagam por seus estudos." Essa afirmação não procede, pois os dados do Exame Nacional de Cursos, que serviram de base para sua análise, neste tópico, mostram o contrário. Contudo, o tipo de dado analisado pelos autores não possibilita inferência desta ordem, pois os dados do Exame Nacional de Cursos referem-se somente aos formandos. Esta limitação enviesa a amostra pois, como se sabe, há uma enorme evasão no sistema privado. Em 2002, por exemplo, esta cifra atingiu 30\%. Sabe-se ainda que uma das principais causas dessa evasão é a incapacidade dos alunos para pagarem as mensalidades no decorrer do curso. Portanto, certamente, entre os concluintes do sistema privado estão os mais "ricos".

Esse mesmo estudo demonstra que o ensino nas instituições públicas é de melhor qualidade, tomando como base o desempenho no Provão. "Com base nos resultados da Prova Geral do Exame Nacional de Cursos, é inegável que o desempenho médio dos formandos egressos das universidades públicas foi superior ao alcançado pelos das demais instituiçóes. Esse resultado mantém-se constante em qualquer que seja o grupo de formandos que se compare. Entre formandos que freqüentaram turnos similares, oriundos de uma mesma região geográfica com renda familiar equivalente, constatou-se sempre que os alunos das universidades públicas têm melhor desempenho que os demais." (p.51)

20 O índice Gini mede o grau de concentração de algum recurso entre membros de uma população determinada. O índice varia de 0 a 1 sendo que 1 representa o máximo de concentração. Esse índice é o mais utilizado para avaliar o grau de concentração de renda nas sociedades. 
Um dos estudos mais sólidos, do ponto de vista metodológico e empírico, sobre a questão do efeito do nível de educação sobre a herança de status socioeconômico na determinação do status ocupacional atual, é o de Michel Hout (1988), realizado nos EUA. De acordo com esse estudo, o efeito de se ter completado o nível superior anula os efeitos do status de origem sobre o status de destino para a população como um todo, reforçando, assim, a tese meritocrática de mobilidade social para as sociedades industrializadas.

Buscando identificar qual a relação do ensino médio e superior com o efeito do status de origem sobre o de destino, tomamos os dados da PNAD nos anos 1973, 1982, 1988 e 1996 (anos em que estava disponível a informação sobre educação superior e origem socioeconômica) e analisamos, através de um modelo de regressão de mínimos-quadrados ordinários, o efeito da herança de status sobre o status de destino. Tomamos como variável dependente o status ocupacional ${ }^{21}$ e como variáveis de controle o nível educacional (ensino médio e ensino superior), sexo, raça e idade, ${ }^{22}$ utilizando como referência para comparação apenas a população que completou a $8^{\underline{a}}$ série do ensino fundamental e que não entrou no ensino médio. Os resultados foram:

Efeitos Independentes do Ensino Médio e do Ensino Superior sobre o Status Socioeconômico dos indivíduos nas PNADs de 1973,1982,1988 e 1996.

21 Essa variável é medida pela escala de 100 pontos construída por Nelson Valle e Silva. Ver Pastore, José e Silva, Nelson do Valle (1999)

22 Esta variável é apresentada de duas formas no modelo: em primeiro lugar ela é incluída como um termo linear e, em segundo lugar, há a inclusão de um termo quadrático. O uso de um termo linear (com valor positivo) e um termo quadrático (com valor negativo) deve-se à relação curvilinear entre idade e o status socioeconômico ocupacional. Expondo essa relação de uma forma mais específica, observa-se que até os 47 anos de idade, essa variável tem um efeito positivo sobre o status socioconômico ocupacional , passando a ter um efeito negativo após este ponto. Além disso, a variável idade foi centralizada para evitar colinearidade, dado que o modelo é polinomial. 
Tabela 3

\begin{tabular}{|c|c|c|c|c|}
\hline $\begin{array}{c}\text { Variáveis } \\
\text { independentes } \\
\text { (controle) }\end{array}$ & 1973 & 1982 & 1988 & 1996 \\
\hline Sexo & $5,93^{*}$ & $2,89^{*}$ & $2,32^{*}$ & $5,54^{*}$ \\
Raça & ---------- & $1,02^{*}$ &, $69(, 001)$ & $1,38^{*}$ \\
Idadecen &, $23^{*}$ &, $174^{*}$ &, $179^{*}$ & $014^{*}$ \\
Idadecen2 &,$- 003^{*}$ &,$- 009^{*}$ &,$- 004^{*}$ &,$- 006^{*}$ \\
EnMédio & $5,71^{*}$ & $6,43^{*}$ & $6,24^{*}$ & $8,82^{*}$ \\
EnSuperior & $18,73^{*}$ & $25,08^{*}$ & $23,99^{*}$ & $23,69^{*}$ \\
locpaicen &, $11^{*}$ &, $10^{*}$ &, $14^{*}$ &, $16^{*}$ \\
Variáveis Teste [i] & & & & \\
locpcenmédio &, $03(, 057)$ &,$- 04(, 004)$ &,$- 05(, 030)$ &,$- 02(, 013)$ \\
locpcensuperior &, $05(, 005)$ &, $06(, 002)$ &, $02(, 319)$ &,$- 01(, 108)$ \\
$\mathrm{N}$ & $17.941,00$ & $25.185,0$ & $19.077,00$ & $48.008,00$ \\
\hline
\end{tabular}

Fonte - PNADS, 1973, 1982, 1988, 1996

Notas:

*os coeficientes com asteriscos indicam que o nível de significância é de $P<0,000$

1- Os números entre parênteses referem-se ao nível de significância

2- Sexo: variável indicativa, $1=$ masc., $\mathrm{o}=$ fem.

3- Raça: variável indicativa, $1=$ branco e amarelo, $0=$ as outras.

4- Idadecen: idade centralizada

5- Idadecen2: idadecentralizada ao quadrado

6- EnMédio: Ensino Médio Completo.

7- EnSuperior: Ensino Superior Completo

8- Iocpaicen: índice socioeconômico centralizado do pai.

9- locpcenmédio: (fator teste) fator interativo do índice ocupacional centralizado do pai educação média.

10- locpcensuperior: (fator teste) fator interativo do índice ocupacional centralizado do pai educação superior.

Como mostram os coeficientes acima, os efeitos independentes do ensino médio e superior sobre o status socioeconômico dos indivíduos seguem o padrão esperado: o efeito do ensino médio tende a crescer entre 1973 e 1996, passando de um efeito que aumenta em 5,70 pontos na escala de status do indivíduo, em 1973, para 8,81 pontos em 1996. O ensino superior passa de um aumento de 18,73 pontos, em 1973, para 
23,69 pontos em 1996, sendo que, em 1982, esta cifra atinge 25,07 (todos esses coeficientes significam um aumento de pontos na escala de status quando comparados com a população que só tem a $8^{\underline{a}}$ série do ensino básico). Quanto ao efeito da interação do status ocupacional do pai e o nível educacional do filho, podemos concluir que:

a) o efeito da interação do status socioeconômico do pai com o nível educacional médio, atua no sentido de reduzir o efeito do status de origem, medido pelo índice ocupacional do pai, sobre o status de destino, medido pelo índice ocupacional do filho. Embora, para a PNAD de 1973 a interação não seja significativa (nos outros anos é significativa a níveis discutíveis considerando o tamanho da amostra), essa tendência é totalmente consistente com o que se poderia esperar, baseando-se nas generalizações empíricas encontradas nos outros países;

b) já no caso do ensino superior a situação é bem diferente. Contrariando a conclusão do estudo de Hout(1988) e as teses meritocráticas em geral das teorias do industrialismo e da modernização, ${ }^{23}$ de que o efeito da educação diminui o efeito do status adscrito nas sociedades moderno-industriais, no caso brasileiro, a obtenção do diploma de nível superior tende a aumentar, ao invés de diminuir o efeito do status de origem sobre o de destino, para os anos de 1973,1982 e 1988 ( ainda que o efeito deste ano não seja significativo). Somente em 1996, inverte-se o sentido desse efeito, mas o valor do coeficiente não é estatisticamente significativo.

Como podemos constatar, vivemos ainda em uma sociedade muito elitizada: nem mesmo o acesso ao ensino superior diminui o efeito do status socioeconômico do pai sobre o status socioeconômico do filho, ao

23 Para uma discussão de excelente qualidade e bastante abrangente sobre essas teorias ver Danielle Fernandes (1997). Em seu estudo empírico no Brasil, tomando seis coortes cobrindo o período de 1832 a 1938, Fernandes conclui que os efeitos meritocráticos do sistema educacional no Brasil são praticamente anulados quando se controla raça. Ver cap. 9, pág. 214 -218. 
contrário, a posse de um certificado de nível superior aumenta a influência do status do pai na definição do status que obterá na estrutura ocupacional. É inegável, à luz destes dados, que a sociedade brasileira ainda, não apenas não quebrou os efeitos do sistema de status adscrito sobre o status ocupacional dos indivíduos, como também reforça os efeitos deste sistema sobre o status ocupacional para o segmento da população com educação superior.

Como explicar este fenômeno aparentemente paradoxal com as características modernas da nossa economia de mercado? Apresentamos aqui uma hipótese para explicar esse fenômeno, combinando duas teorias contemporâneas sobre a alocação de indivíduos na estrutura ocupacional. A primeira, de M. Granovetter $(1974,1995)$ sugere que as redes sociais constituem um fator crucial para a alocação de indivíduos na estrutura ocupacional. ${ }^{24}$ A segunda, de P. Bourdieu (1986) propõe que o capital social dos indivíduos é um forte determinante de suas chances de mobilidade na estrutura ocupacional. O conceito de capital social de P. Bourdieu refere-se "à soma dos recursos, reais ou virtuais, a disposição de um indivíduo ou grupo, em virtude da existência de uma rede de relacionamentos, mais ou menos institucionalizada, de mútuo entendimento e reconhecimento." (p. 248-9). À luz destas teorias, os dados acima podem ser interpretados como evidência de que as redes de relações sociais, no Brasil, são fortemente relacionadas à herança de status e funcionam como um tipo de capital social que favorece a aquisição do status de destino.

De qualquer forma, é surpreendente que um dos canais de mobilidade social, tipicamente meritocrático, e dos mais visíveis em qualquer sociedade contemporânea, a educação superior, funcione na sociedade brasileira como um fator que reforça a sua face mais oligárquica e estamental, ainda nos dias de hoje.

24 Essa teoria recebeu suporte empírico pela pesquisa do próprio Granovetter no iníco dos anos 70, relatada no livro Getting a Job-A Study of Contacts And Careers, Chicago: University of Chicago Press, 1995 ( 2 a edition). 


\section{Conclusão}

Nosso artigo sugere que a expansão do canal de acesso ao ensino superior, em todo o mundo, embora tenha apresentado efeitos significativos no plano da democratização do acesso, não foi suficiente para derrubar a muralha de classe social, resguardando o privilégio de entrada nas grandes e tradicionais universidades em quase todos os países do planeta. O caso brasileiro corrobora esta ten dência mundial, embora com feições diferentes. Em primeiro lugar, o sistema privado de ensino constitui o "equivalente funcional" do sistema terciário não universitário da Europa e dos EUA, na medida em que foi sua expansão que aumentou o acesso aos setores menos privilegiados da sociedade brasileira, e não o sistema público, que continuou fortemente elitista. Em segundo lugar, distintamente do que ocorre no contexto mundial, o título universitário, aqui, aumenta o efeito particularista da origem de classe sobre o destino ocupacional das pessoas, anulando, assim, o efeito do mérito na mobilidade ocupacional.

\section{Referências}

AMARAL, A. and TEIXEIRA, Pedro, "The Rise and Fall of the Private Sector in Portuguese Higher education". Higher Education Policy. 13 (2000) 245-266.

BOURDIEU, Pierre. "The forms of capital" In: RICHARDSON, J. G. (org.) Handbook for Theory and Research for the Sociology of Education. Westport: Greenwood, p. 241-258, 1986.

BOUDON, R. A Desigualdade de Oportunidades. Brasíla: Editora da Universidade de Brasília, 1981.

CORBUCl, P. R., As Universidades Federais: gastos, desempenho, eficiência e produtividade. Textos para Discussão No, 752. Brasíla(DF): Instituto de Pesquisa Aplicada (IPEA). 2000. 
FERNANDES. D. Educational Stratification, Race and Socioeconomic Development in Brazil. Tese (Ph.D Sociologia), Madison: University of Wisconsin.1999.

GRANOVETTER, M., "Economic Action and Social Structure: the problem of embeddedness. In: GRANOVETTER, Mark, Getting a Job. Appendix D. Chicago and London: The University of Chicago Press ( $2^{\text {nd }}$ edition). 1995.

HOUT, M., "More Universalism, Less Structural Mobility: The American Occupational Structure in the 1980s". American Journal of Sociology, 93 no. 6 (May 1988):1358-1400.

JARUSCH, H.K, "Higher Education and Social Change: some comparative perspectives". In: JARUSCH, H. Konrad. (org), The Transformation of Higher Education 1860-1930. Chicago: University of Chicago Press. 1983:9-36.

KERR, C. Os Usos da Universidade, Fortaleza: universidade federal do Ceará, 1982.

KRYMKOSWSKI, H., D., " The Process of Status Attainment Among men in Poland, The U.S., and West Germany". American Sociological Review, 56 (February 1991): 46-59.MEEK, V.L., "Diversity and Marketization of Higher Education: imcompatible concepts?". Higher Education Policy, 13 (2000) 23-49.

NEAVE, G. "Diversity, Differentiation and the Market: the debate we never had but which we ought to have done." Higher education Policy, 13 (2000): 7-21.

OSBORNE, M., " Increasing or Widening Participation in Higher Education? - a European overview." European Journal of Education. 38, no. 1 (2003):5-24.

PASTORE, J. e VALLE Silva N., Mobilidade Social no Brasil. Makron Books, 1999 RYAN, P. "Evaluating Vocationalism". European Journal of Education, 38, no.2 (2003):147-162.

SAMPAIO H., Limongi, F. e TORRES H. Equidade e Heterogeneidade no Ensino Superior Brasileiro. Brasília, DF: INEP. 2000.

SHAVIT, Y. and H.P. Blossfeld, (eds), persistent inequality: Changing Educational Attainment in Thirteen Countries, Bouldr, CO: Westview Press, 1993.

SCHWARZTMAN, S. O Ensino Superior no Brasil. Brasília: MEC/INEP.1998. 
TROW, M., "Reflections on the tnasition from mass to universal higher education", Daedalus, 99, 1970, 1-42.

TROW, M. On mass higher educationand institutional diversification. Tel Aviv: Samuel Neaman Institute for Advanced Studies in Science and technology. May, 2003.

The International Bank for Reconstruction and Development/World Bank, Higher Education: the lessons of experience.Washington. 1994.

UNESCO, World Statistical Outlook on Higher Education 1980-1995. Paris: 1998.

Organization For Economic Co-Operation and Development (OECD). Redefining Tertiary Education. Paris. 1998.

\section{Resumo}

Este trabalho pretende mostrar como a enorme expansão do sistema de ensino superior no âmbito mundial, desde os anos 70, resulta da incorporação da lógica mercantil no sistema de gestão pública do ensino superior. Este traço das políticas públicas que tem caracterizado a grande maioria dos sistemas de gestão de ensino superior nas sociedades contemporâneas tem, entretanto, permitido o insulamento das universidades de prestígio acadêmico destas políticas, mantendo-as como nichos exclusivos dos membros das elites sociais destas sociedades. A análise do caso brasileiro mostra um quadro ainda mais dramático do que o internacional, pois aqui, o credenciamento superior aumenta, ao invés de diminuir, os efeitos do status de origem sobre o status socioeconômico (SSE).

Palavras-chave: Sistema de Ensino Superior, teceirização, desigualdade, universidade, status socioeconômico, política educacional, modelo institucional.

Recebido: 18/12/06

Aceite final: $15 / 02 / 07$ 


\section{Universities VS outsourcing of higher education - the logic of broadening access while keeping inequality: the case of Brazil}

\section{Antônio Augusto Pereira Prates}

This work intends to demonstrate how the large expansion in the higher education system all over the world since the 1970 s results in the incorporation of market logic in the system of public management for higher education. However, such feature of public policies that has marked the vast majority of systems for management of higher education in contemporary societies has allowed isolating universities that enjoy academic reputation from those policies, keeping them as exclusive niches for members of social elites. The analysis of the Brazilian case shows an even more dramatic picture than the international one, since here, having higher education increases rather than reducing the effects of original status on social-economic status.

Key words: Higher education system, Outsourcing, Inequality, University, Socialeconomic status, Educational policy, Institutional model. 